\title{
A double mutation in families with periodic paralysis defines new aspects of sodium channel slow inactivation
}

\author{
Saïd Bendahhou, ${ }^{1}$ Theodore R. Cummins, ${ }^{2,3}$ Angelika F. Hahn, ${ }^{4}$ Sylvie Langlois, ${ }^{5}$ \\ Stephen G. Waxman, ${ }^{2,3}$ and Louis J. Ptácek ${ }^{1,6,7}$ \\ ${ }^{1}$ Howard Hughes Medical Institute, Eccles Institute of Human Genetics, University of Utah, Salt Lake City, Utah, USA \\ ${ }^{2}$ Department of Neurology, Yale University School of Medicine, New Haven, Connecticut, USA \\ ${ }^{3}$ Department of Neurology, Paralyzed Veterans of America/Eastern Paralyzed Veterans of America Neuroscience \\ Research Center, Veterans Affairs Medical Center, West Haven, Connecticut, USA \\ ${ }^{4}$ University of Western Ontario, Department of Clinical Neurological Sciences, London, Ontario, Canada \\ ${ }^{5}$ Department of Medical Genetics, British Columbia's Children Hospital, Provincial Medical Genetics Programme, \\ Vancouver, British Columbia, Canada \\ ${ }^{6}$ Department of Neurology, and \\ ${ }^{7}$ Department of Human Genetics, University of Utah, Salt Lake City, Utah, USA
}

Address correspondence to: Louis J. Ptácek, Howard Hughes Medical Institute, University of Utah,

Building 533, Room 4420, 15 North 2030 East, Salt Lake City, Utah 84112, USA.

Phone: (801) 581-3993; Fax: (801) 585-5597; E-mail: ptacek@howard.genetics.utah.edu.

Received for publication February 21, 2000, and accepted in revised form June 13, 2000.

\begin{abstract}
Hyperkalemic periodic paralysis (HyperKPP) is an autosomal dominant skeletal muscle disorder caused by single mutations in the $S C N 4 A$ gene, encoding the human skeletal muscle voltage-gated $\mathrm{Na}^{+}$channel. We have now identified one allele with two novel mutations occurring simultaneously in the SCN4A gene. These mutations are found in two distinct families that had symptoms of periodic paralysis and malignant hyperthermia susceptibility. The two nucleotide transitions predict phenylalanine $1490 \rightarrow$ leucine and methionine $1493 \rightarrow$ isoleucine changes located in the transmembrane segment $\mathrm{S} 5$ in the fourth repeat of the $\alpha$-subunit $\mathrm{Na}^{+}$channel. Surprisingly, this mutation did not affect fast inactivation parameters. The only defect produced by the double mutant (F1490L-M1493I, expressed in human embryonic kidney 293 cells) is an enhancement of slow inactivation, a unique behavior not seen in the 24 other disease-causing mutations. The behavior observed in these mutant channels demonstrates that manifestation of HyperKPP does not necessarily require disruption of slow inactivation. Our findings may also shed light on the molecular determinants and mechanism of $\mathrm{Na}^{+}$channel slow inactivation and help clarify the relationship between $\mathrm{Na}^{+}$channel defects and the long-term paralytic attacks experienced by patients with HyperKPP.
\end{abstract}

J. Clin. Invest. 106:431-438 (2000).

\section{Introduction}

Voltage-gated ion channels represent a large family that includes $\mathrm{K}^{+}, \mathrm{Ca}^{2+}$, and $\mathrm{Na}^{+}$channels, and they are associated with a variety of diseases such as long QT syndrome, Brugada's syndrome, hypokalemic periodic paralysis, hyperkalemic periodic paralysis (Hyper$\mathrm{KPP}$ ), paramyotonia congenita (PC), and potassiumaggravated myotonia (PAM) (for review, see refs. 1, 2). More than 20 missense mutations have been identified in the human skeletal muscle $\mathrm{Na}^{+}$channel gene causing either HyperKPP, PC, or PAM. Symptoms in patients with PC include muscle stiffness and episodes of weakness induced by exposure to cold. Although PC is characterized by cold-induced hypercontraction of muscles, PAM occurs after ingestion of $\mathrm{K}^{+}$-rich food. Patients with the HyperKPP disorder typically show episodic loss of excitability of skeletal muscle that occurs after intense exercise, exposure to cold or after ingestion of $\mathrm{K}^{+}$-rich food. All the mutations previously reported have been single missense mutations. The defect in $\mathrm{Na}^{+}$channel function induced by these mutations is generally attributed to an abnormal persistent inward $\mathrm{Na}^{+}$current.

Five different HyperKPP mutations (T704M, A1156T, M1360V, I1495F, and M1592V) have been identified in the muscle $\mathrm{Na}^{+}$channel gene. The attacks of weakness can be precipitated by potassium administration or by periods of vigorous activity (3-8). According to the predicted structure and the arrangement of the $\mathrm{Na}^{+}$channel $\alpha$-subunit (9), these amino acids are thought to be located in the intracellular face of the membrane or in the S5 segment. Although none of these HyperKPP mutations were reported to occur in the interdomain III-IV, which appears to be strongly implicated in $\mathrm{Na}^{+}$channel inactivation, these mutations cause a partial disruption of the inactivation properties, suggesting a possible implication of these residues in the inactivation process $(8,10-12)$.

Both fast and slow inactivation have been identified in skeletal muscle $\mathrm{Na}^{+}$channels. Slow inactivation is substantially impaired by some HyperKPP mutations 
$(8,13,14)$. However, it has been recently shown that some of the skeletal muscle $\mathrm{Na}^{+}$channel mutations lead to an enhancement of slow inactivation $(8,15)$. Little is known about $\mathrm{Na}^{+}$channel slow inactivation (e.g., molecular mechanism), and its role in $\mathrm{Na}^{+}$channel function is not well understood.

In the present study, we report the first $\mathrm{Na}^{+}$channel $\alpha$-subunit double mutation that causes HyperKPP. The behavior of the double mutant channels is unique because it produces no persistent current and no apparent shift in either inactivation or activation curves. However, the major defect observed in the double mutant channels is an enhancement of slow inactivation. These two mutations occur in the putative S5 segment in the fourth domain of the $\mathrm{Na}^{+}$channel. We have recently reported a mutation in the S5 segment of domain IV, which also causes HyperKPP (8). Interestingly, this mutation also enhances $\mathrm{Na}^{+}$channel slow inactivation. The present study defines the role of new amino acids in IVS5 of the $\mathrm{Na}^{+}$that are involved in channel slow inactivation.

\section{Methods}

Genetic analysis. Genomic DNA was extracted from peripheral blood leukocytes and amplified using the PCR as described previously (16). Single strand conformation polymorphism (SSCP) analysis was performed on amplified DNA using a protocol described previously (16).

Cell culture and transfection. Human embryonic kidney (HEK) 293 cells were grown and maintained as described previously (8) in $100-\mathrm{mm}$ culture dishes at $37^{\circ} \mathrm{C}$ under $5 \% \mathrm{CO}_{2}$. The calcium-phosphate precipitation technique (17) was used for both transient and stable transfection.

Construction of hSkM1, F1490L, M1493I, and F1490LM1493I mutants. The amino acids located in the S5 segment of domain IV of the human skeletal muscle $\mathrm{Na}^{+}$ channel (hSkM1) were altered using the megaprimer PCR method of site-directed mutagenesis (18) as described previously (8). Primers used for first- and second-round PCR are summarized in Table 1 . The protocol for the first round of PCR was: 2 minutes at $94^{\circ} \mathrm{C}$ for one cycle; 20 seconds at $94^{\circ} \mathrm{C}, 20$ seconds at $50^{\circ} \mathrm{C}$, and 1 minute at $75^{\circ} \mathrm{C}$ for 30 cycles; and 5 minutes at $72^{\circ} \mathrm{C}$ for one cycle. The following protocol was applied during the second round of PCR: 2 minutes at $94^{\circ} \mathrm{C}$ for one cycle, 20 seconds at $94^{\circ} \mathrm{C}, 20$ seconds at $54^{\circ} \mathrm{C}$, and 90 seconds at $72^{\circ} \mathrm{C}$ for 30 cycles; and 5 minutes at $72^{\circ} \mathrm{C}$ for one cycle. All PCR reactions were carried out in DNA Engine Tetrad (MJ Research, Watertown, Massachusetts, USA). DNA sequencing was performed using either ABI dRhodamine dye terminators, or ABI Prism BigDye Terminators and cycle sequencing with Taq FS DNA polymerase. DNA sequence was collected and analyzed on an ABI Prism 377 automated DNA sequencer (Perkin-Elmer Applied Biosystems, Foster City, California, USA). Primers were synthesized on a 394 automated DNA synthesizer (Perkin-Elmer Applied Biosystems) following standard ABI procedures.

Electrophysiology. Recordings were conducted in the whole-cell configuration (19) at room temperature $\left(22^{\circ} \mathrm{C}\right)$, as described previously $(8)$. Twenty-four hours after plating stable lines or 36 hours after transfection, culture media was replaced with the bathing solution: $140 \mathrm{mM} \mathrm{NaCl}, 4 \mathrm{mM} \mathrm{MgCl}, 2 \mathrm{mM} \mathrm{CaCl}_{2}$, and $10 \mathrm{mM} \mathrm{Na-HEPES} \mathrm{(pH} \mathrm{7.3).} \mathrm{The} \mathrm{internal} \mathrm{pipette}$ solution contained: $130 \mathrm{mM} \mathrm{CsCl,} 4 \mathrm{mM} \mathrm{MgCl}_{2}, 2.5$ mM EGTA, $5 \mathrm{mM} \mathrm{NaCl}$, and $10 \mathrm{mM}$ HEPES ( $\mathrm{pH}$ 7.3). In the experiments examining slow inactivation, $\mathrm{CsF}$ was substituted for $\mathrm{CsCl}$ to improve the long-term stability of the recordings.

\section{Results}

Evaluation of patients. We report here a new patient with HyperKPP in kindred 3744 and a family (kindred 2514) from which clinical data were published earlier (20). The proband in $\mathrm{K} 3744$ is a 32-year-old man who has experienced episodic attacks of paralysis and weakness since age 15 years. The attacks were not worsened with cold or provocative maneuvers including fasting and rest. The patient evaluation included an electromyogram, without cooling, which showed myotonic discharges (data not shown). He usually wakes up with either partial or generalized paralysis that involves limbs and usually resolves over a period of 3-5 days. He occasionally experiences a sense of weakness that may last for several weeks. Clinically, the patient has very little myotonia, other than intermittent lid lag, very transient tongue myotonia, and myotonic contraction of the finger extensors and thenar muscles. Muscle biopsy analysis showed typical tubular aggregates representing sarcolemmal and T-tubular changes (1). The patient's 56year-old mother is known to have had paralytic attacks, which seem to be less frequent with age. Her motor examination was remarkable for eyelid myotonia. Even with cooling, myotonia was not elicited in the hands.
Table 1

Primers used in PCR reactions to engineer F1490L, M1493I, and F1490L-M1493I mutant constructs

\begin{tabular}{|c|c|c|}
\hline Mutation & $\begin{array}{l}\text { First-round PCR primers } \\
\qquad\left(5^{\prime} \text { to } 3^{\prime}\right)\end{array}$ & $\begin{array}{l}\text { Second-round PCR primers } \\
\left(5^{\prime} \text { to } 3^{\prime}\right)\end{array}$ \\
\hline $\begin{array}{l}\mathrm{F} 1490 \mathrm{~L} \\
(\mathrm{~T} 4545 \mathrm{C})\end{array}$ & $\begin{array}{l}\text { GGCTCAATGTCAAGGTCAAC } \\
\text { TCCTCCTCCTCCTGGTCATG } \\
\text { CATGACCAGGAGGAGGAGGA } \\
\text { TGGGCAAGTCCAGTGTGATG }\end{array}$ & $\begin{array}{c}\text { GGCTCAATGTCAAGGTCAAC } \\
\text { TGGGCAAGTCCAGTGTGATG } \\
\text { and two megaprimers from first round } \\
\text { PCR }\end{array}$ \\
\hline $\begin{array}{l}\text { M1493I } \\
(\mathrm{G} 4556 \mathrm{~A})\end{array}$ & $\begin{array}{l}\text { GGCTCAATGTCAAGGTCAAC } \\
\text { CCTGGTCATATTCATCTACTC } \\
\text { GAGTAGATGAATATACCAGG } \\
\text { TGGGCAAGTCCAGTGTGATG }\end{array}$ & $\begin{array}{c}\text { GGCTCAATGTCAAGGTCAAC } \\
\text { TGGGCAAGTCCAGTGTGATG } \\
\text { and two megaprimers from first round } \\
\text { PCR }\end{array}$ \\
\hline $\begin{array}{l}\text { F1490L- } \\
\text { M1493I } \\
\text { (T4545C, } \\
\text { G4556A) }\end{array}$ & $\begin{array}{l}\text { GGCTCAATGTCAAGGTCAAC } \\
\text { TGGGCAAGTCCAGTGTGATG }\end{array}$ & $\begin{array}{c}\text { GGCTCAATGTCAAGGTCAAC } \\
\text { TGGGCAAGTCCAGTGTGATG } \\
\text { and two megaprimers from first round } \\
\text { PCR }\end{array}$ \\
\hline
\end{tabular}


Remaining tests of her motor function were normal. Needle electromyography was performed on the right biceps brachii, first dorsal interosseous, and tibialis anterior muscles. Insertional activity was increased, and the patient demonstrated florid myotonic potentials in all muscles tested. She has had two paralytic attacks, at the age of 35 years, both occurring after a general anesthesia, suggesting a particular sensitivity to anesthetics that can trigger an attack in this family. A similar phenotype has been reported in patients described previously (20) with both HyperKPP and MH.

Identification of nucleotide alterations in the patients' DNA. DNA from our patient and from patients described previously (20) was amplified using primers for SCN4A gene as described elsewhere (8). An aberrant SSCP conformer was noted with primers designed to amplify exon 24. Sequence analysis of the aberrant conformer revealed T4545C and G4556A mutations (data not shown) of the SCN $4 A$ gene that predict phenylalanine $\rightarrow$ leucine and methionine $\rightarrow$ isoleucine changes at positions 1490 and 1493, respectively, in one allele of the human skeletal muscle $\mathrm{Na}^{+}$channel $\alpha$-subunit.
These mutations were absent in DNA from more than 100 unrelated normal individuals. These two amino acids are located in the membrane-spanning segment S5 of domain IV, and are well-conserved among all voltage-gated $\mathrm{Na}^{+}$channels sequenced to date from Drosophila to human (Figure 1).

Inactivation and activation. Four cDNA constructs (wild-type [WT], F1490L, M1493I, and F1490LM1493I) were introduced into the $\mathrm{pRc-CMV}$ vector heterologously expressed in HEK 293 cells, and studied using the whole-cell voltage clamp technique. When compared with WT macroscopic current, the mutant channels exhibited similar fast activation and inactivation kinetics (Figure 2). There was no sustained current observed after 25-millisecond depolarization.

Closed-state inactivation was assessed in all four channels. Figure 3 shows that whereas the F1490L mutant shifts the steady-state inactivation to the left $\left(\mathrm{V}_{1 / 2}=\right.$ $-66.3 \pm 0.85 \mathrm{mV}, n=29, P<0.001)$, M1493I shifts the inactivation curve to the right $\left(\mathrm{V}_{1 / 2}=-56.6 \pm 1.0 \mathrm{mV}, n\right.$ $=19, P<0.001)$ compared with the WT $\left(\mathrm{V}_{1 / 2}=-62.1 \pm\right.$ $0.75 \mathrm{mV}, n=43)$. However, the inactivation curve for the

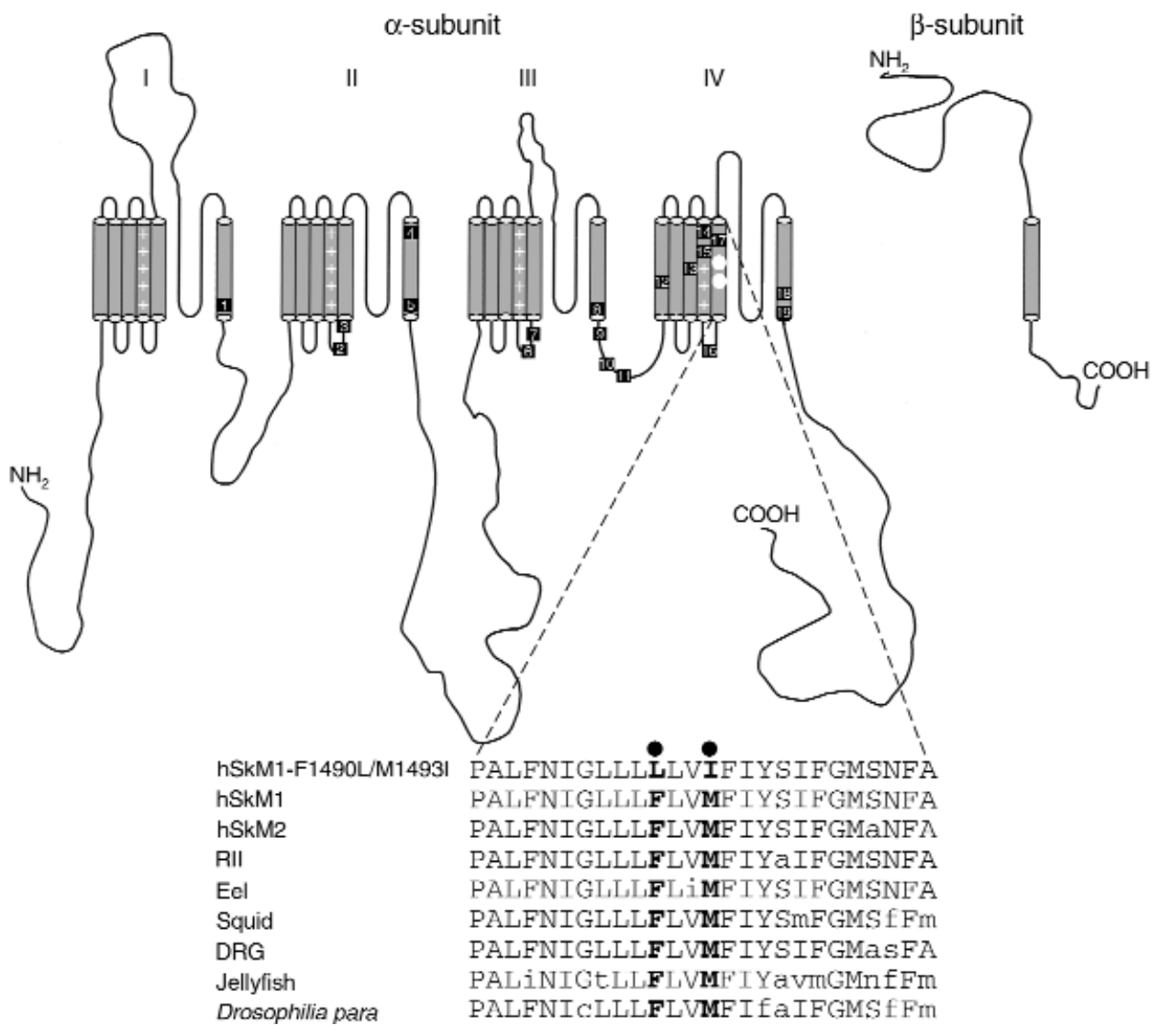

Figure 1

Schematic of the voltage-gated skeletal muscle $\mathrm{Na}^{+}$channel $\alpha$-subunit showing the location of naturally occurring mutations described to date (mutations 1-19) and the double mutation (open circles) F1490L-M1493I described in this study. Mutations 1, 4, 6, 7, 8, and 18 are associated with PAM; mutations $2,9,12,13,14,15$, and 16 are associated with PC; and mutations $3,5,10,11,17$, and 19 are associated with HyperKPP. The lower panel shows how the phenylalanine and methionine mutated are well conserved between different cloned $\mathrm{Na}^{+}$ channels: human skeletal muscle (hSkM1), human cardiac muscle (hSkM2), rat brain type II (RII), eel electroplax, squid giant axon, TTXresistant $\mathrm{Na}^{+}$channels from dorsal root ganglia (DRG), jellyfish, and Drosophila para. 
WT

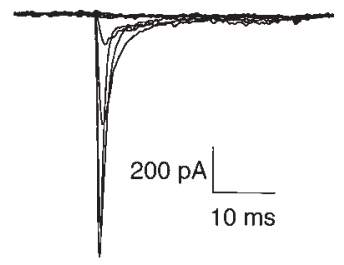

F1490L

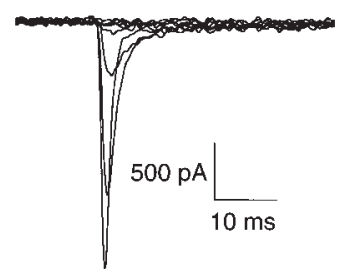

Figure 2

Current traces from representative HEK 293 cells expressing either WT channels, F1490L, M1493I, or F1490L-M1493I mutant channels. $\mathrm{Na}^{+}$ currents were monitored in the whole-cell configuration and were elicited by repetitive 25 -millisecond voltage steps ranging from -80 to $0 \mathrm{mV}$. The holding potential was $-120 \mathrm{mV}$. pA, picoamperes.

double mutant $\left(\mathrm{V}_{1 / 2}=-62.5 \pm 0.82 \mathrm{mV}, n=34, P=0.7\right)$ is indistinguishable from that of WT. The activation curves were affected in a different manner: the F1490L mutation slightly, but not significantly, shifted the activation curve to the left $\left(\mathrm{V}_{1 / 2}=-19.1 \pm 0.65 \mathrm{mV}, n=28, P\right.$ $=0.08$ ), and the M1493I shifted the activation curve to the right $\left(\mathrm{V}_{1 / 2}=-13.0 \pm 0.89 \mathrm{mV}, n=21, P<0.001\right)$. However, the double mutant F1490L-M1493I slightly shifted the midpoint of activation to hyperpolarizing potentials $\left(\mathrm{WTV}_{1 / 2}=-17.7 \pm 0.44 \mathrm{mV}, n=47 ; \mathrm{F} 1490 \mathrm{~L}-\mathrm{M} 1493 \mathrm{I}\right.$ $\left.\mathrm{V}_{1 / 2}=-20.1 \pm 0.62 \mathrm{mV}, n=24, P<0.003\right)$. When combining the inactivation and activation curves, none of the mutations are predicted to significantly increase window currents in agreement with our observations on the macroscopic currents for all channels at the potentials tested. The behavior of the double mutation is

\section{Figure 3}

Activation and inactivation curves. Peak $\mathrm{Na}^{+}$conductance $\left(G_{\mathrm{Na}}\right)$ was measured during a 25-millisecond depolarization to various test potentials from a holding potential of $-120 \mathrm{mV}$ to characterize steady-state activation for WT (filled circles; $n=47$ ), F1490L (filled diamonds; $n=28$ ), M1493I (filled squares; $n=21$ ), and double mutant F1490L-M1493I (open circles; $n=24$ ). $G_{\mathrm{Na}}$ is calculated from the relation $G_{\mathrm{Na}}=I_{\mathrm{Na}}\left(V-V_{\mathrm{rev}}\right)$, where $I_{\mathrm{Na}}$ is the peak inward $\mathrm{Na}^{+}$current during the test depolarization $(V)$ and $V_{\text {rev }}$ is the $\mathrm{Na}^{+}$reversal potential. Data are normalized to maximum peak conductance $\left(G_{\max }\right)$ and fit to a two-state Boltzmann distribution: $G_{N a} / G_{\max }=(1+$ $\left.\exp \left[\left(V-V_{1 / 2}\right) / k\right]\right)^{-1}$, where $V_{1 / 2}$ is the test potential for half-maximal $\mathrm{Na}^{+}$activation and $\mathrm{k}$ determines the steepness of the voltage dependence. Inactivation curves plotted for $\mathrm{Na}^{+}$currents for WT (filled circles; $n=43$ ), F1490L (filled diamonds; $n=29$ ), M1493I (filled squares; $n=19$ ), and double mutant F1490L-M1493I (open circles; $n=34$ ). Cells were held at $-120 \mathrm{mV}$ and subjected to a 200 -millisecond conditioning pulse ranging from -120 to $0 \mathrm{mV}$ followed by a 25 millisecond test pulse to $0 \mathrm{mV}$. Values are mean $\pm \mathrm{SEM}$. unique, as no disease-causing mutation to date has been described with such a behavior. To our knowledge at least one of the parameters listed here has been affected in all disease-causing mutations described to date.

Deactivation and recovery from fast inactivation. To investigate further how HyperKPP can occur in families with the S5 segment double mutation, we investigated deactivation and recovery from fast inactivation in all four channels. Recovery from fast inactivation showed no difference between WT and the three mutant channels (Figure 4a). It has been recently hypothesized that, in addition to a defect in channel inactivation, deactivation can contribute to the disease $(21,22)$. Deactivation time constants were monitored at voltages ranging from $-120 \mathrm{mV}$ to $-60 \mathrm{mV}$ and revealed no significant change between $\mathrm{WT}$ and either the single mutants F1490L and M1493I or the double mutant channels (Figure 4b).

Enhancement of slow inactivation in HyperKPP mutations. It has been reported that a defect in slow inactivation may be an important factor in the maintenance of paralysis in some patients with HyperKPP (23). Hence, we investigated slow inactivation in the four channels. Figure 5a shows that slow inactivation was significantly enhanced in F1490L mutant channels $\left(\mathrm{s}_{1 / 2}=\right.$ $-73.1 \pm 2.5 \mathrm{mV}, n=9, P<0.005)$ and in the double mutant channels $\left(\mathrm{s}_{1 / 2}=-73.8 \pm 1.9 \mathrm{mV}, n=18, P<\right.$ $0.05)$, but slightly shifted to the right for the M1493I mutant channels $\left(\mathrm{s}_{1 / 2}=-62.3 \pm 2.1 \mathrm{mV}, n=9, P=0.23\right)$. The midpoint of slow inactivation for WT channels was $-65.9 \pm 1.7 \mathrm{mV}(n=23)$. Enhanced slow inactivation can have important consequences on excitability. As Figure 5b illustrates, although only 50\% of WT channels are slow inactivated when held at $-60 \mathrm{mV}$ (for 50 seconds), almost $90 \%$ of F1490L-M1493I channels are slow inactivated at the same holding potential. The enhanced slow inactivation seen in the double mutant can dramatically reduce the number of functional channels. We also compared the time course for the development of, and recovery from, slow inactivation

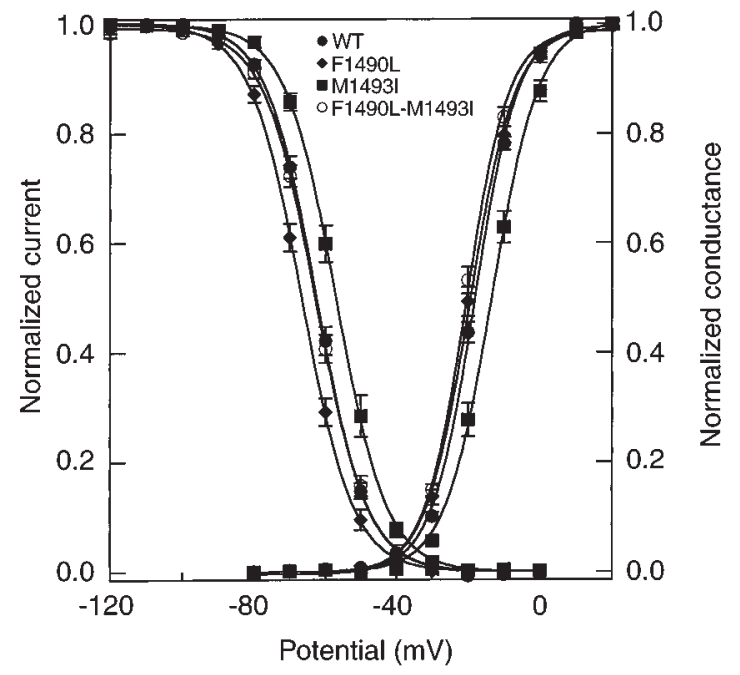


for WT and the double mutant channels. Development of slow inactivation was examined at $-50 \mathrm{mV}$ (Figure 5c). The time constant for development of slow inactivation was similar for WT $(6.2 \pm 0.7$ seconds, $n=$ $4)$ and double mutant $(6.4 \pm 0.6$ seconds, $n=4)$ channels. However, after 2 minutes at $-50 \mathrm{mV}$, significantly more double mutant channels are slow inactivated $(89 \pm 1 \%, n=4, P<0.005)$ than WT channels $(72 \pm 3 \%$, $n=4)$. Recovery from slow inactivation, on the other hand, was slower for double mutant and F1490L channels than for WT channels (Figure 5d). Recovery was measured at $-100 \mathrm{mV}$ after 5 minutes at $0 \mathrm{mV}$. After 1 second, significantly more WT channels $(38 \pm 4 \%, n=$ 9) than double mutant channels $(16 \pm 2 \%, n=8, P<$ 0.001 ) are available for activation. If the time course for recovery from slow inactivation is fit with a dual exponential function, the first time constant is significantly larger for double mutant channels $(4.3 \pm 0.6$ seconds, $n=8, P<0.0002)$ than for WT channels $(1.5 \pm$ 0.2 seconds, $n=9)$, but the second time constant is similar $(32.6 \pm 1.6$ seconds vs. $33.6 \pm 4.2$ seconds $)$. Recovery from slow inactivation was also examined at $-80 \mathrm{mV}$ for WT and double mutant channels (data not shown). As shown by Hayward et al. (1997) (14), recovery from slow inactivation is roughly 10 fold slower at $-80 \mathrm{mV}$ than at $-100 \mathrm{mV}$ for WT channels expressed in HEK 293 cells. Recovery from inactivation was also slower for double mutant channels at $-80 \mathrm{mV}$, and after 10 seconds, significantly more WT channels than double mutant channels are available for activation.

Does the double mutation affect the interaction between $\alpha$ and $\beta$ subunits? The voltage-gated $\mathrm{Na}^{+}$channel consists of $\alpha$ and $\beta$ subunits in skeletal muscle. The role of the $\beta$ subunit was shown to affect channel inactivation when coinjected in oocytes. However, it has little effect on the kinetics of fast inactivation when coexpressed with $\alpha$ in HEK 293 cells. A depolarizing shift in the inactivation curve due to $\beta_{1}$ was reported (24). Studies have localized the interactions between $\alpha$ and $\beta_{1}$ subunits to the loop between S5 and S6 transmembrane segments (25). The location of the double amino acid substitution in the S5 segment makes it appealing to look for potential interactions between $\beta_{1}$ subunit and the S5 segment. We cotransfected HEK 293 cells with $\alpha$ (hSkM1-WT, F1490L, M1493I, or double mutant F1490L-M1493I) and $\beta_{1}$ (from brain). These experiments did not show any significant alteration of the interaction between $\alpha$ and $\beta_{1}$ subunits (Table 2). Slow inactivation was not altered by the presence of $\beta_{1}$ subunit. No significant change was observed in the recovery from fast inactivation, or in the deactivation time constant between the WT coexpressed with $\beta_{1}$ and the other mutant channels co-expressed also with $\beta_{1}$ (data not shown).

\section{Discussion}

The gating behavior of single mutants (F1490L and M1493I) and the double mutant channels (F1490LM1493I) was investigated in a mammalian cell line to understand the cause of the disease in two affected fam- ilies carrying this double mutation. Compared with the 24 single mutations studied so far (Figure 1), the behavior of the double mutant channels expressed in HEK 293 cells was unique in several respects: no persistent $\mathrm{Na}^{+}$current was observed after a 100-millisecond or even a 25-millisecond test depolarization; no shift was observed in either activation or inactivation curves, thus no increase in window current was recorded; and no major change in deactivation time constant was observed. The double mutant channels apparently exhibit only an enhancement of slow inactivation compared with WT channels (Figure 5). There are two mechanisms by which $\mathrm{Na}^{+}$channels can inactivate: fast and slow. Fast inactivation has an onset and recovery on the order of milliseconds, whereas slow inactivation develops and recovers on the scale of seconds or even minutes. Although the structural basis for fast inacti-
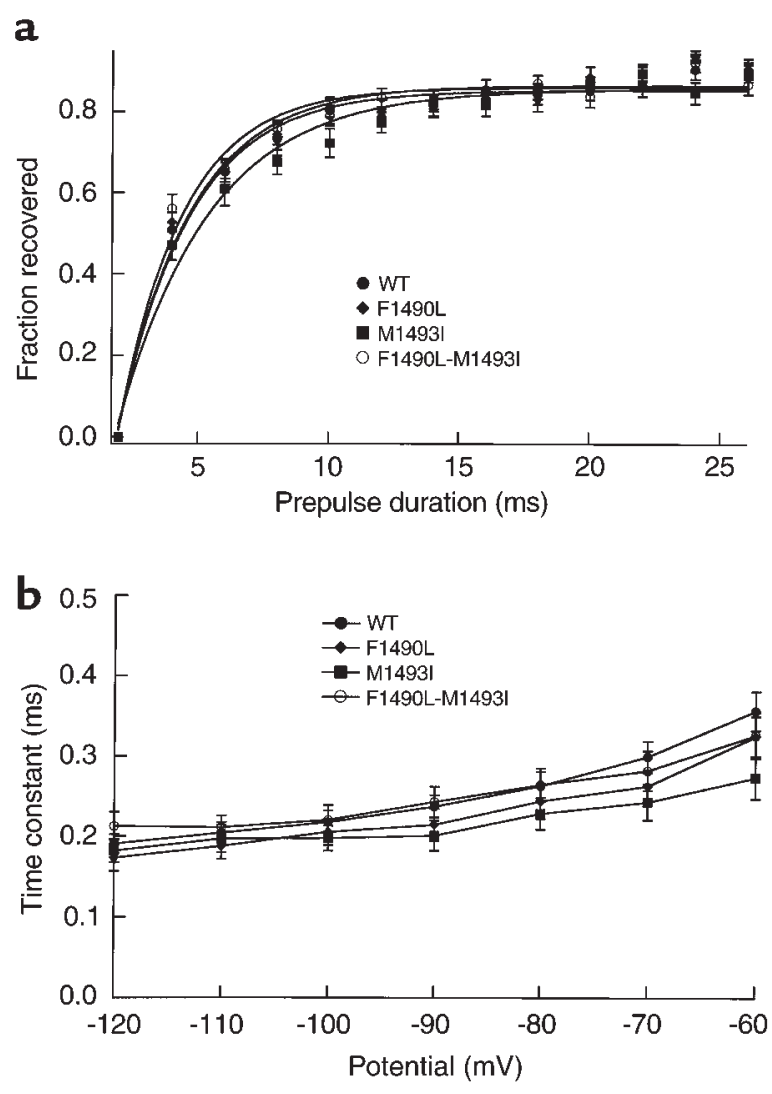

\section{Figure 4}

Recovery from fast inactivation and deactivation. (a) Recovery from fast inactivation. Cells were prepulsed to $0 \mathrm{mV}$ for 200 milliseconds to inactivate all of the current, then recovery potentials from -120 to $-60 \mathrm{mV}(-120 \mathrm{mV}$ is shown) for increasing recovery duration were applied before the test pulse to $0 \mathrm{mV}$ (20 milliseconds) to assay the fraction of current recovered. Traces obtained at the test pulse were fitted to a single-exponential function. (b) Tail currents were elicited by a 0.5 -millisecond test pulse to $+40 \mathrm{mV}$ followed by a repolarization pulse ranging from -120 to $-60 \mathrm{mV}$. Resulting currents were fitted by a single-exponential decay and expressed as a function of the voltage for WT (filled circles; $n=20$ ), F1490L (filled diamonds; $n=20$ ), M1493I (filled squares; $n=20$ ), and F1490L-M1493I (open circles; $n$ $=20)$. Values represent mean \pm SEM. 
Table 2

Effect of $\beta_{1}$ subunit on channel activation, fast and slow inactivation

\begin{tabular}{llll}
\hline Transfection & \multicolumn{1}{c}{$\begin{array}{c}\text { Activation } \\
\mathrm{V}_{1 / 2}(\mathrm{mV})\end{array}$} & $\begin{array}{c}\text { Fast inactivation } \\
\mathrm{V}_{1 / 2}(\mathrm{mV})\end{array}$ & $\begin{array}{c}\text { Slow inactivation } \\
\mathrm{s}_{1 / 2}(\mathrm{mV})\end{array}$ \\
$\mathrm{WT}$ & $-17.7 \pm 0.4 ; n=47$ & $-62.1 \pm 0.7 ; n=43$ & $-65.9 \pm 1.7 ; n=23$ \\
$\mathrm{WT}+\beta_{1}$ & $-15.7 \pm 0.8 ; n=10$ & $-65.0 \pm 1.1 ; n=10$ & $-61.0 \pm 4.5 ; n=6$ \\
F1490L-M1493I & $-20.1 \pm 0.6 ; n=24$ & $-62.5 \pm 0.8 ; n=34$ & $-73.8 \pm 1.9 ; n=18$ \\
$\mathrm{~F} 1490 \mathrm{~L}-\mathrm{M} 1493 \mathrm{I}+\beta_{1}$ & $-14.2 \pm 0.8 ; n=11$ & $-63.4 \pm 1.9 ; n=11$ & $-68.4 \pm 2.8 ; n=9$ \\
$\mathrm{~F} 1490 \mathrm{~L}$ & $-19.1 \pm 0.6 ; n=28$ & $-66.3 \pm 0.8 ; n=29$ & $-73.1 \pm 2.5 ; \mathrm{n}=9$ \\
F1490L $+\beta_{1}$ & $-18.5 \pm 0.8 ; n=7$ & $-70.0 \pm 1.9 ; n=7$ & $\mathrm{ND}$ \\
$\mathrm{M} 1493 \mathrm{I}$ & $-13.0 \pm 0.9 ; n=21$ & $-56.6 \pm 1.0 ; n=19$ & $-62.3 \pm 2.1 ; n=9$ \\
$\mathrm{M} 1493 \mathrm{I}+\beta_{1}$ & $-15.2 \pm 1.6 ; n=6$ & $-65.1 \pm 2.6 ; n=6$ & $\mathrm{ND}$ \\
\hline
\end{tabular}

ND, not done.

vation is known, the molecular entity involved in $\mathrm{Na}^{+}$ channel slow inactivation is still under investigation. Studies involving disease-causing mutations have indicated that the S4-S5 linker $(13,14,26)$, S5 (8), S6 (15, 27) segments, and the pore region (28) may be involved in slow inactivation.

Muscle $\mathrm{Na}^{+}$channel diseases are often functionally linked to defects in channel fast inactivation. The kinetics of fast inactivation were unaffected in F1490L, M1493I, and F1490L-M1493I macroscopic $\mathrm{Na}^{+}$currents when compared with WT at all voltages tested. The steady-state fast inactivation curve, on the other hand, was shifted to hyperpolarizing potentials in the F1490L mutant channels, and exhibited a depolarizing shift in the M1493I channels. However, the two single mutants apparently canceled each other's effects in the double mutant, because the steady-state fast inactivation curve for the double mutant was nearly identical to that of WT channels. No defect was seen in the time constants of deactivation for all mutant channels studied here, ruling out the possibility of the contribution of channel deactivation in the disease as previously postulated for some of the PC Na${ }^{+}$channel mutants (21, 22). Our patients showed variable myotonia. It is not clear how myotonia developed without alterations of activation, fast inactivation, or deactivation. This may be due to limitations of our expression system. The variable nature of myotonia in our patients suggests that genetics and environmental factors contribute to the expression of this mutation. Studying biopsied muscle patients in vitro may shed the light on the mechanism underlying myotonia in patients with the double mutation in segment S5.

A defect in channel slow inactivation has been postulated to be an important factor in the development and maintenance of paralysis that is associated with some $\mathrm{Na}^{+}$channel diseases, such as HyperKPP (23). Cummins and Sigworth (13) have shown that slow inactivation underlies HyperKPP in rT698M, the rat homologue of the most common human HyperKPP mutation (T704M), and we have recently shown the same behavior in human T704M channels (8). Other groups have reported a disruption of slow inactivation in some of the other HyperKPP mutations $(14,26)$.
When slow inactivation was investigated in our mutants, F1490L and F1490LM1493I exhibited an enhancement of slow inactivation shown by a hyperpolarizing shift in the steady-state slow inactivation (Figure 5). However, the M1493I mutant channels slightly shifted the slow inactivation curve in the depolarizing direction. We have recently reported a mutation in the S5 segment of domain IV, I1495F, which also causes HyperKPP (8). Interestingly, the $11495 \mathrm{~F}$ mutation also enhances $\mathrm{Na}^{+}$channel slow inactivation. However, in contrast to F1490L-M1493I, which apparently affects only slow inactivation, $14495 \mathrm{~F}$ altered fast inactivation and the voltage-dependence of activation in addition to its effect on slow inactivation. Our studies suggest that the S5 segment of domain IV is important in $\mathrm{Na}^{+}$channel slow inactivation. Coexpression of $\alpha$ subunit with $\beta_{1}$ did not lead to alteration of the mutant channel behavior despite the fact that $\beta_{1}$ was shown to modulate slow inactivation of the hSkM1 channels in oocytes (29). However, $\beta_{1}$ may modulate other aspects of hSkM1 such as protein trafficking. If the double mutant targets this type of interaction, it would have been missed in the HEK 293 expression system. Additionally, the channel kinetics and voltage dependencies in HEK 293 cells may be different from those found for channels in native tissue and at physiological temperature (30).

How could missense mutations cause paralysis with only an enhancement of slow inactivation? In normal muscle, slow inactivation would be expected to occur in depolarized cells, shutting down the long depolarizing $\mathrm{Na}^{+}$currents and repolarizing myocyte membranes. When the cell membrane is depolarized for a long period, the enhanced slow inactivation leads to functional loss of channels, and consequently to weakness from which it takes a long time to recover. In the double mutant channels, slow inactivation was enhanced and was more complete. This enhancement is due to a faster development of slow inactivation and a slower recovery from slow inactivation, which could result in a longer refractory period that may lead to paralysis. Recently, Takahashi and Cannon (15) reported that a mutation in the S6 segment of domain I that is associated with myotonia, but not weakness, also enhances slow inactivation. The manifestations of HyperKPP in our patients showed myotonia, suggesting that an enhancement of slow inactivation is not sufficient to prevent myotonia in HyperKPP patients.

Although HEK 293 cells have been widely used for the study of $\mathrm{Na}^{+}$channel mutations associated with myotonic disorders, the channel kinetics and voltage dependencies in HEK 293 cells may exhibit important differences from those found for channels in native tissue. For example, although the midpoint of slow 
inactivation is about $-65 \mathrm{mV}$ for WT SkM1 channels expressed in HEK 293 cells (this study and refs. 13, $14)$, it is around $-105 \mathrm{mV}$ in rat skeletal muscle (31, 32). Recovery from slow inactivation also appears to be one to two orders of magnitude slower for sodium currents in skeletal muscle (30) than for hSkM1 channels expressed in HEK 293 cells. Thus, it might be expected that the time constants for recovery from slow inactivation of the double mutant channels will be significantly longer in native muscle than the values reported in this study for channels expressed in HEK 293 cells. However, caution must be used when extrapolating from studies done at room temperature on channels expressed in HEK 293 cells to channels in native tissue. Although the kinetics of slow inactivation might be much slower for WT channels in native muscle, the kinetics are approximately fivefold faster at $37^{\circ} \mathrm{C}$ than at room temperature, and the voltage dependence of slow inactivation is shifted in the depolarizing direction by approximately $15 \mathrm{mV}$ (30). Furthermore, as has been proposed by Ruff (30), some

$\mathbf{a}$

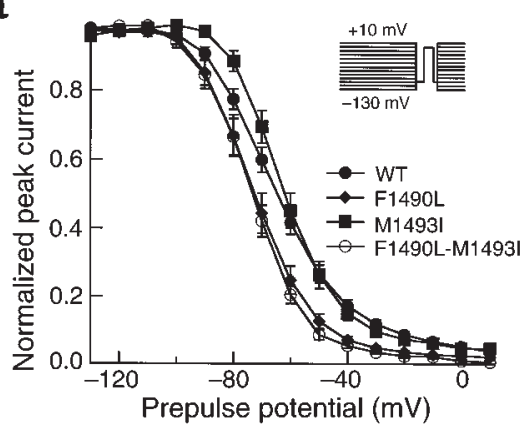

C

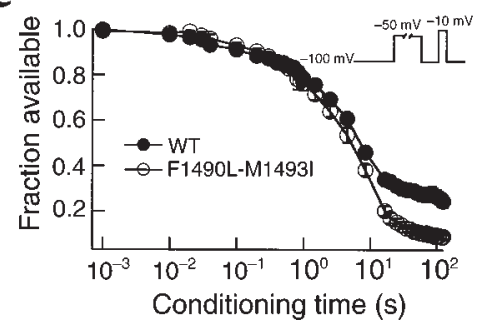

of the sodium channel disease mutations might alter the effects of temperature on the kinetics or voltage dependence of slow inactivation. Studying mutant sodium channels expressed in muscle tissue at physiological temperatures may be helpful to develop a fuller understanding of the mechanisms underlying myotonia and paralysis in patients with specific disease mutations.

Taken together with our recent study on the I1495F (8) located in the S5 segment of domain IV, the present observations strongly support the idea that the S5 segment of domain IV is involved in channel slow inactivation. Because we believe that the part of the $\mathrm{Na}^{+}$channel that governs slow inactivation may not be confined in one (or two) molecular site as it is for fast inactivation, we suggest that conformational arrangements of several parts of the $\mathrm{Na}^{+}$channel $\alpha$-subunit such as segments S5, S6, and the pore region from different domains underlie the mechanism by which voltage-gated $\mathrm{Na}^{+}$channels may undergo slow inactivation.

b
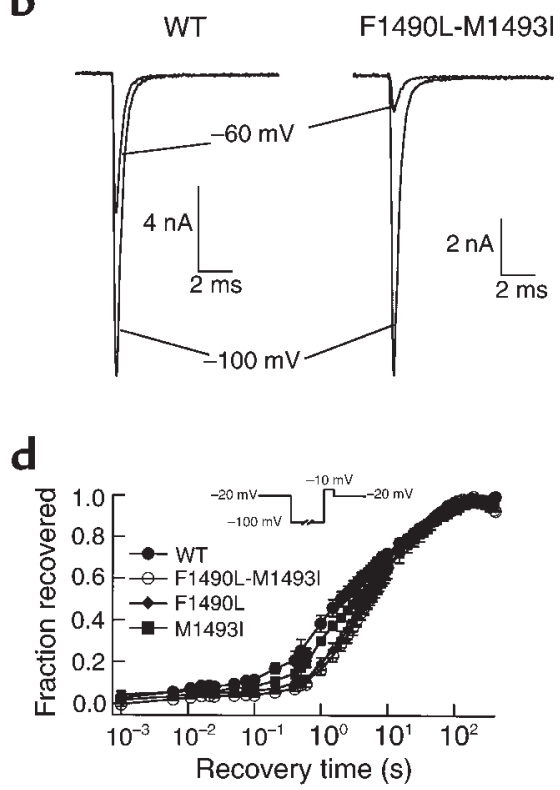

\section{Figure 5}

Enhancement of slow inactivation in the F1490L-M1493I channels. (a) Steady-state slow inactivation in hSkM1 (filled circles; $n=23$ ), F1490L (filled diamonds; $n=9$ ), M1493I (filled squares; $n=9$ ), and F1490L-M1493I (open circles; $n=18$ ) channels. The first step consists of holding the cells at potentials ranging from -130 to $10 \mathrm{mV}$ in 10-mV steps for 50 seconds. A 30-millisecond recovery pulse to -100 $\mathrm{mV}$ and a 20 -millisecond test pulse to $-10 \mathrm{mV}$ were given before the holding potential was incremented again. The holding potential was incremented by $10 \mathrm{mV}$ immediately after each recovery pulse/test pulse sequence. We term this protocol sequential because the channels are not allowed to recover from slow inactivation for each test pulse; the expectation is that the sequential changes in holding potential will mimic changes in holding potential of longer durations. The short hyperpolarizing recovery pulses can be used to remove fast inactivation just before a test pulse to measure slow inactivation (31). The peak current elicited by the test pulse to $-10 \mathrm{mV}$ was plotted as a fraction of the maximum current. (b) A greater fraction of F1490L-M1493I current than WT current is slow inactivated at $-60 \mathrm{mV}$. Cells were held for 50 seconds at $-60 \mathrm{mV}$, allowed to recover for 30 millisecond at $-100 \mathrm{mV}$, and then depolarized to $-10 \mathrm{mV}$ to determine the fraction of channels that are slow inactivated. For comparison, the total current available from a holding potential of $-100 \mathrm{mV}$ is also shown for WT and F1490L-M1493I channels. (c) Development of slow inactivation is shown to be faster for the F1490L-M1493I (open circles; $n=5$ ) than for the WT channels (filled circles; $n=5$ ). Cells were held at $-100 \mathrm{mV}$ for an increasing conditioning time. (d) Normalized $\mathrm{Na}^{+}$current in representative WT (filled circles; $n=9$ ), F1490L (filled diamonds; $n=8$ ), M1493I (filled squares; $n=7$ ), and F1490L-M1493I (open circles; $n=8$ ) cells recovering from slow inactivation (conditioning pulse is $-50 \mathrm{mV}$ ). The time axis is logarithmic. The recovery protocol required about 45 minutes to complete and had two phases: short recovery times were obtained with individual recovery pulses, and long recovery times were obtained in a continuous recording. 


\section{Acknowledgments}

The authors thank J. Richmond, D. Featherstone, and M. Sheets for critical reading of the manuscript. This work was supported by a Muscular Dystrophy Association grant (LP), a Public Health Service Research grant M01RR000064 from the National Center for Research Resources, and by the Paralyzed Veterans of America and Eastern Paralyzed Veterans Association. We also thank the DNA Sequencing Facility at the University of Utah, supported in part by National Cancer Institute grant 5P30CA42014.

1.Ptácek, L.J., and Griggs, R.C. 1996. Familial periodic paralysis. Plenum Press. New York, New York, USA. 625-642.

2. Bulman, D.E. 1997. Phenotype variation and newcomers in ion channel disorders. Hum. Mol. Genet. 10:1679-1685.

3. Ptácek, L.J., et al. 1991. Identification of a mutation in the gene causing hyperkalemic periodic paralysis. Cell. 67:1021-1027.

4. Rojas, C.V., et al. 1991. A Met-to-Val mutation in the skeletal muscle $\mathrm{Na}^{+}$ channel $\alpha$-subunit in hyperkalaemic periodic paralysis. Nature. 354:387-389.

5. McClatchey, A.I., et al. 1992. Novel mutations in families with unusual and variable disorders of skeletal muscle sodium channel. Nat. Genet. 2:148-152.

6. Ptácek, L.J., et al. 1993. Sodium channel mutations in paramyotonia congenita and hyperkalemic periodic paralysis. Ann. Neurol. 33:300-307.

7. Lehmann, H.F., Rudel, R., and Ricker, K. 1993. Non-dystrophic myotonias and periodic paralyses. A European Neuromuscular Center Workshop held 4-6 October 1992, Ulm, Germany. Neuromuscul. Disord. 3:161-168.

8. Bendahhou, S., Cummins, T.R., Tawil, R., Waxman, S.G., and Ptácek, L.J. 1999. Activation and inactivation of the voltage-gated sodium channel: role of segment S5 revealed by a novel hyperkalaemic periodic paralysis mutation. J. Neurosci. 19:4762-4771.

9. Noda, M., et al. 1984. Primary structure of Electrophorus electricus sodium channel deduced from cDNA sequence. Nature. 312:121-127.

10. Cannon, S.C., and Strittmatter, S.M. 1993. Functional expression of sodium channel mutations identified in families with periodic paralysis. Neuron. 10:317-326.

11. Yang, N., et al. 1994. Sodium channel mutations in paramyotonia congenita exhibit similar biophysical phenotypes in vitro. Proc. Natl. Acad. Sci. USA. 91:12785-12789.

12. Wagner, S., et al. 1997. A novel sodium channel mutation causing a hyperkalemic paralytic and paramyotonic syndrome with variable clinical expressivity. Neurology. 49:1018-1025.

13. Cummins, T.R., and Sigworth, F.J. 1996. Impaired slow inactivation in mutant sodium channels. Biophys. J. 71:227-236.

14. Hayward, L.J., Brown, R.H., Jr., and Cannon, S.C. 1997. Slow inactivation differs among mutant $\mathrm{Na}$ channels associated with myotonia and peri- odic paralysis. Biophys. J. 72:1204-1219.

15. Takahashi, M.P., and Cannon, S.C. 1999. Enhanced slow inactivation by V445M: a sodium channel mutation associated with myotonia. Biophys. J. 76:861-868.

16. Ptácek, L.J., et al. 1992. Mutations in an S4 segment of the adult skeletal muscle sodium channel cause paramyotonia congenita. Neuron. 8:891-897.

17. Graham, F.L., and Van Der Eb, A.J. 1973. A new technique for assay for infectivity of human adenovirus 5 DNA. Virology. 52:456-467.

18. Sarkar, G., and Sommer, S.S. 1990. The "megaprimer" method of sitedirected mutagenesis. Biotechniques. 8:404-407.

19. Hamill, O.P., Marty, A., Neher, E., Sakmann, B., and Sigworth, F.J. 1981. Improved patch-clamp techniques for high-resolution current recording from cells and cell-free membrane patches. Pflugers Arch. 391:85-100.

20. Moslehi, R., Langlois, S., Yam, I., and Friedman, J.M. 1998. Linkage of malignant hyperthermia and hyperkalemic periodic paralysis to the adult skeletal muscle sodium channel (SCN4A) gene in a large pedigree. Am. J. Med. Genet. 76:21-27.

21. Richmond, J.E., VanDeCarr, D., Featherstone, D.E., George, A.L.J., and Ruben, P.C. 1997. Defective fast inactivation recovery and deactivation account for sodium channel myotonia in the I1160V mutant. Biophys. J. 73:1896-1903.

22. Featherstone, D.E., Fujimoto, E., and Ruben, P.C. 1998. A defect in skeletal muscle sodium channel deactivation exacerbates hyperexcitability in human paramyotonia congenita. J. Physiol. (Lond). 506:627-638.

23. Ruff, R.L. 1994. Slow $\mathrm{Na}^{+}$channel inactivation must be disrupted to evoke prolonged depolarization-induced paralysis. Biophys. J. 66:542-545.

24. Bendahhou, S., Cummins, T.R., Potts, J.F., Tong, J., and Agnew, W.S. 1995. Serine-1321-independent regulation of the $\mu 1$ adult skeletal muscle $\mathrm{Na}^{+}$channel by protein kinase C. Proc. Natl. Acad. Sci. USA. 92:12003-12007.

25. Makita, N., Bennett, P.B., and George, A.L. 1996. Multiple domains contribute to the distinct inactivation properties of human heart and skeletal muscle $\mathrm{Na}^{+}$channels. Circ. Res. 78:244-252.

26. Hayward, L.J., Sandoval, G.M., and Cannon, S.C. 1999. Defective slow inactivation of sodium channels contributes to familial periodic paralysis. Neurology. 52:1447-1453.

27. Wang, S.Y., and Wang, G.K. 1997. A mutation in segment I-S6 alters slow inactivation of sodium channels. Biophys. J. 72:1633-1640.

28. Balser, J.R., et al. 1996. External pore residue mediates slow inactivation in $\mathrm{mu} 1$ rat skeletal muscle sodium channels. J. Physiol. (Lond.) 494:431-442.

29. Vilin, Y.Y., Makita, N., George, A.L., Jr., and Ruben, P.C. 1999. Structural determinants of slow inactivation in human cardiac and skeletal muscle sodium channels. Biophys. J. 77:1384-1393.

30. Ruff, R.L. 1999. Effects of temperature on slow and fast inactivation of rat skeletal muscle $\mathrm{Na}\left({ }^{+}\right)$channels. Am. J. Physiol. 277:C937-C947.

31. Simoncini, L., and Stühmer, W. 1987. Slow sodium channel inactivation in rat fast-twitch muscle. J. Physiol. (Lond.) 383:327-337.

32. Ruff, R.L., Simoncini, L., and Stühmer, W. 1987. Comparison between slow sodium channel inactivation in rat slow- and fast-twitch muscle. J. Physiol. (Lond.) 383:339-348. 